\title{
Combined treatment of XIAP-targeting shRNA and celecoxib synergistically inhibits the tumor growth of non-small cell lung cancer cells in vitro and in vivo
}

\author{
HONG ZHANG, ZHIHONG LI, KAIZHONG WANG and PING REN \\ Department of Thoracic Surgery, The First Hospital of Jilin University, \\ Chaoyang, Changchun, Jilin 130021, P.R. China
}

Received May 29, 2014; Accepted July 16, 2014

DOI: $10.3892 /$ or.2014.3678

\begin{abstract}
X-linked inhibitor of apoptosis protein (XIAP) has been shown to be highly expressed in lung cancer, but not in normal lung tissue, which makes it an attractive target for lung cancer treatment. Celecoxib (CXB), a cyclooxygenase-2 inhibitor, is in wide clinical use for the treatment and prevention of non-small cell lung cancer (NSCLC). Therefore, in our study, we combined short hairpin RNA (shRNA) targeted to XIAP (XIAP-shRNA) with CXB and tested the effects of this combination on lung cancer cells to identify more effective therapeutics against lung cancer. An XIAP-shRNA plasmid was constructed and transfected into the A549 NSCLC cell line. The cells were then treated with CXB and XIAP-shRNA alone or in combination for indicated time periods, and the treatments were assessed for their effects on cell proliferation, apoptosis, migration, invasion and receptor signaling using the MTT, TUNEL, wound healing and Matrigel invasion assays and western blotting, respectively. In addition, an NSCLC xenograft model was prepared to observe tumor growth. It was found that both CXB and XIAP-shRNA significantly inhibited cell proliferation, migration and invasion, and induced cell apoptosis in vitro, as well as suppressed tumor growth in vivo. Moreover, the combination of the agents significantly enhanced these effects compared to the single agent treatments. We also found that the combination treatment significantly suppressed constitutive phosphorylation of PI3K and AKT, which may contribute to the inhibition of tumor growth. These findings suggest that the combination of XIAP-shRNA and CXB is a promising drug candidate for the treatment of NSCLC.
\end{abstract}

Correspondence to: Professor Ping Ren, Department of Thoracic Surgery, The First Hospital of Jilin University, 71 Xinming Street, Chaoyang, Changchun, Jilin 130021, P.R. China

E-mail: renping14428@sina.com

Key words: lung cancer, non-small cell lung cancer, X-linked inhibitor of apoptosis protein, short hairpin RNA, celecoxib

\section{Introduction}

Lung cancer is one of the most common neoplasms worldwide, and approximately $85-90 \%$ of lung cancer cases are non-small cell lung cancer (NSCLC) (1). Despite the fact that chemotherapy and radiotherapy have been widely used in the treatment of advanced NSCLC, the outcome remains poor, with less than $15 \%$ of patients surviving beyond 5 years due to the lack of early diagnosis and effective treatment methods $(2,3)$. Therefore, it is urgent to identify new therapeutics to combat this disease.

With the recent advances in the understanding of the molecular pathways involved in NSCLC progression, targeted therapies that are designed to interfere with the mechanisms of cancer cell growth and survival offer new hope in NSCLC therapeutics. X-linked inhibitor of apoptosis protein (XIAP) has been identified as one of the most potent inhibitors of caspases and apoptosis to date and is known to play an important role in cell apoptosis, cell migration and cell invasion $(4,5)$. It is highly expressed in various malignancies, while its expression is very low or absent in normal cells, which makes it an attractive target for cancer therapeutics (6-8). A large number of studies have demonstrated that inhibition of XIAP expression using antisense oligonucleotides or small interfering RNA (siRNA) could suppress the proliferation of tumor cells, induce cell apoptosis, and sensitize tumor cells to chemotherapeutic agents (9-14). In addition, Harlin et al found that XIAP-knockout mice have normal survival with no significant pathological features, consistent with XIAPtargeted therapeutics exerting minimal toxicity to normal tissues (15). Therefore, in the present study, we explored the possibility of XIAP as a therapeutic target for the treatment of human NSCLC.

Celecoxib (CXB), a selective cyclooxygenase-2 (COX-2) inhibitor, has been used in the treatment of a wide range of cancers including NSCLC $(2,16)$. Previous studies have shown that the antitumor mechanisms of $\mathrm{CXB}$ include different pathways, such as the death receptor, mitochondria-mediated pathways, Akt phosphorylation inhibition, endoplasmic reticulum (ER) stress, and autophagy (17-21). Despite its excellent antitumor activity, CXB has a relatively low therapeutic index, and its clinical usage is limited due to acute and chronic 
toxicities such as cardiovascular thrombosis, congestive heart failure, gastrointestinal ulceration, renal or hepatic injury and platelet aggregation (22). In addition, resistance to this agent is common, representing a major obstacle to successful treatment. The toxicity of doxorubicin can be reduced if it is used in conjunction with another, more tumor-specific treatment, in order to reduce the dosage. Currently, CXB is widely being tested in clinical trials for its therapeutic activity against various cancers in combination with other agents (23-25). For example, Morisaki et al found that the combination of sorafenib (SOR) and CXB has synergistic anti-proliferative and pro-apoptotic effects on human liver cancer cells (25). Our recent study also showed that $\mathrm{CXB}$ in combination with SOR suppressed VEGFR and COX-2 expression and inhibited the tumor growth of NSCLC (3). Huang et al demonstrated that downregulation of glucose-regulated protein (GRP) 78 potentiated by RNAi enhanced CXB-induced cell apoptosis in human urothelial carcinoma cells (26). These findings suggest that CXB in combination with other agents could allow a decrease in the dose of the drug, and increase antitumor activity.

In the present study, we aimed to investigate the efficacy of a combination of XIAP-targeted short hairpin RNA (XIAP-shRNA) and CXB in the treatment of NSCLC in vitro and in vivo on the basis of the hypothesis that this combination exhibits a synergistic anticancer effect compared with either treatment alone. We found that this combination treatment led to high antitumor activity in vitro and in vivo. This combination is a promising drug candidate for the treatment of NSCLC.

\section{Materials and methods}

Reagents and antibodies. $\mathrm{CXB}$, one type of COX-2 inhibitor, was purchased from Pfizer Corporation Inc. (New York, NY, USA) and was dissolved in dimethyl sulfoxide (DMSO). 3-(4,5-Dimethylthiazol-2-yl)-2,5-diphenyltetrazolium bromide (MTT) was obtained from Sigma Aldrich (St. Louis, MO, USA), and was prepared by dissolving $1 \mathrm{mg}$ of each compound in $1 \mathrm{ml}$ of phosphate-buffered saline (PBS, pH 7.2). For western blot analysis, the following antibodies were used: mouse monoclonal anti- $\beta$-actin (Sigma Aldrich), mouse monoclonal anti-Bcl-2, mouse monoclonal anti-survivin, mouse monoclonal anti-PI3K, mouse monoclonal anti-phosphorylated (p)-PI3K, mouse monoclonal anti-AKT, mouse monoclonal anti-p-AKT and anti-mouse IgG were purchased from Santa Cruz Biotechnology (Santa Cruz, CA, USA).

shRNA design and plasmid construction. The cDNA sequence of XIAP was obtained from GenBank (accession number NM-001167). The siRNA target design tools from Ambion were used to design XIAP-shRNA, and non-specific shRNA sequences were designed according to the siRNA target design tools from Ambion (Austin, TX, USA). XIAPshRNA and non-specific shRNA were designed and synthesized as follows: XIAP-shRNA sense, 5'-GAT CCGGTGAAGGTGATAAAGTAATCAAGAGATACAAA TTCTTCTTCCTTAAGA-3' and antisense, 5'-AGCTTCT GATCCGGTGAAGGTGATAAAGTAACTCTTGAATACA AATTCTTCCTTAG-3'; control shRNA sense, 5'-GATCCT
TCTCCGAACGTGTCACGTTCAAGAGAAAACTACTTC TTTTACCTTAGA-3' and antisense, 5'-AGCTTCTTCTTT CTCCGAACGTGTCACGTCTCTTGAAAAACTACTTCT TTTACCTTG-3'; the sequence does not target any gene product and has no significant sequence similarity to human gene sequences. The oligonucleotides were annealed and then ligated into the BamHI and HindIII sites of pSilencer 4.1-CMV neo expression vector according to the manufacturer's instructions (Ambion). The recombinant vectors were confirmed by the digestion analysis of restriction endonuclease, and all inserted sequences were verified by DNA sequencing. Each vector contained the SV40 early promoter to provide G418 resistance in mammalian cells. The shRNA insert template oligonucleotides encoding XIAP are referred to as XIAP-shRNA, and the plasmid encoding non-specific siRNA, which was used as a negative control, is referred to as NC-shRNA.

Cell culture and transfection. The human NSCLC cell line A549 was purchased from the Cell Bank of the Type Culture Collection of the Chinese Academy of Sciences, Shanghai Institute of Cell Biology, Chinese Academy of Sciences (Shanghai, China). A549 cells were cultured in RPMI-1640 medium (Invitrogen, Carlsbad, CA, USA) supplemented with heat-inactivated $10 \%$ fetal bovine serum (FBS; Biochrom AG) at $37^{\circ} \mathrm{C}$ in a humidified atmosphere containing $5 \% \mathrm{CO}_{2}$.

A549 cells were seeded in 6-well plates at $2.0 \times 10^{4}$ cells $/$ well, respectively and cultured overnight to $80 \%$ confluency prior to transfection. Transfection was performed using Lipofectamine Plus (Grand Island, NY, USA), and the ratio of the plasmids and the transfection reagent was $1 \mathrm{mg}: 2 \mathrm{ml}$. Cells were transfected with the different plasmids (XIAP-shRNA, NC-shRNA) according to the protocol specifications. G418 $(800 \mu \mathrm{g} / \mathrm{ml}$, Sigma Aldrich) was used to screen stably transfected clones. Stable transfectants were named A549/XIAP-shRNA and A549/NC-shRNA.

Quantification by real-time polymerase chain reaction. Total cellular RNA was isolated using TRIzol reagent according to the manufacturer's protocol (Invitrogen). M-MLV reverse transcriptase (Fermentas MBI, Amherst, NY, USA) was used to create cDNA following the manufacturer's instructions. Quantitative real-time polymerase chain reaction (RT-PCR) assays were carried out using SYBR-Taq real-time kits (Takara Biotechnology, Otsu, Japan) and RT-PCR amplification equipment ABI Prism 7900HT (Applied Biosystems, Foster City, CA, USA). The PCR primers used to detect XIAP and GAPDH were as follows: XIAP forward, 5'-GACAGTATGCAAGAT GAGTCAAGTCA-3' and reverse, 5'-GCAAAGCTTCTCCTC TTGCAG-3', with a product length of $93 \mathrm{bp}$; GAPDH forward, 5'-TGTGGGCATCAATGGATTTGG-3' and reverse, 5'-ACA CCATGTATTCCGGGTCAAT-3', with a product length of $171 \mathrm{bp}$. The PCR conditions were as follows: pre-denaturation at $94^{\circ} \mathrm{C}$ for $5 \mathrm{~min}$, followed by 40 cycles of denaturation at $94^{\circ} \mathrm{C}$ for $10 \mathrm{sec}$, annealing/extension at $58^{\circ} \mathrm{C}$ for $15 \mathrm{sec}$ and a final extension at $72^{\circ} \mathrm{C}$ for $10 \mathrm{~min}$. The amplification specificity was checked by melting curve analysis. The expression of the genes of interest was determined by normalization of the threshold cycle $(\mathrm{Ct})$ of these genes to that of the control 
GAPDH. The $2^{-\Delta \Delta C t}$ method was used to calculate the relative abundance of target gene expression generated using RotorGene Real-Time analysis software 6.1.81.

Western blot analysis. The A549 cells were then homogenized in a lysis buffer (Tris- $\mathrm{HCl} 50 \mathrm{mmol} / \mathrm{l}$, EDTA $5 \mathrm{mmol} / \mathrm{l}, \mathrm{NaCl}$ $150 \mathrm{mmol} / \mathrm{l}$, sodium deoxycholate $1 \%, \mathrm{Na}_{3} \mathrm{VO}_{4} 500 \mu \mathrm{mol} / \mathrm{l}$, Triton X-100 0.5\%, AEBSF $10 \mu \mathrm{mol} / \mathrm{l}, \mathrm{NaF} 10 \mathrm{mmol} / \mathrm{l}$ ) on ice. The homogenates were then centrifuged at $14,000 \mathrm{rpm}$ at $4^{\circ} \mathrm{C}$ for $30 \mathrm{~min}$, and the supernatants were collected for protein concentration determination using the BCA assay kit (Sigma Aldrich). Cell extracts (50 $\mu \mathrm{g}$ of protein) were separated on a sodium dodecyl sulfate-polyacrylamide electrophoretic gel (SDS-PAGE) and transferred to nitrocellulose membranes, which were blocked in $3 \%$ bovine serum albumin (BSA) for $2 \mathrm{~h}$. After blocking, the membranes were incubated with the primary antibodies overnight at $4^{\circ} \mathrm{C}$ for $2 \mathrm{~h}$, and subseqnently with the horseradish peroxidase-conjugated secondary antibody for $2 \mathrm{~h}$ at room temperature. Protein bands were visualized with enhanced chimioluminescence reagent (ECL; Amersham, GE Healthcare, Velizy-Villacoublay, France). Densitometry was performed by Quantity One image analysis software.

Cell viability assay. To measure the effect of shRNA-XIAP in combination with CXB on cell proliferation, an MTT assay was used. The cell density of A549 cells was adjusted to $5 \times 10^{4} / \mathrm{ml}$, and cells were added to a 96-well plate (100 $\mu \mathrm{l} /$ well). In the blank controls, $100 \mu \mathrm{l}$ of medium alone was added. At $24 \mathrm{~h}$ after culture, cells were treated with the indicated concentrations of CXB and transfected with the XIAP-shRNA plasmid, or both. Cells were divided into five groups: normal control, negative control (NC-shRNA), XIAP-shRNA group, CXB group and XIAP-shRNA combined with CXB group. There were 8 wells in each group/subgroup. At $48 \mathrm{~h}$ after culture, $200 \mu \mathrm{l}$ of MTT $(5 \mathrm{mg} / \mathrm{ml})$ was added to each well followed by incubation at $37^{\circ} \mathrm{C}$ for $4 \mathrm{~h}$. Then, centrifugation was performed at $2,000 \mathrm{x}$ g for $10 \mathrm{~min}$. The supernatant was removed, and $200 \mu \mathrm{l}$ of DMSO was added to each well followed by shaking for $10 \mathrm{~min}$. Absorbance was measured at $490 \mathrm{~nm}$ with a microplate reader (Molecular Devices Corp., Sunnyvale, CA, USA), and growth inhibition was calculated. The mean proliferation of cells without any treatment was expressed as $100 \%$. All experiments were performed in triplicate and repeated at least three times.

TUNEL assay. To measure the effect of XIAP-shRNA in combination with $\mathrm{CXB}$ on cell apoptosis, TUNEL assay was carried out. In briefly, cellular DNA fragmentation was measured with the ApoTag Red In Situ Apoptosis Detection kit (Chemicon Inc., Temecula, CA, USA) according to the manufacturer's instructions when A549 cells were treated with XIAP-shRNA, CXB alone or both for $24 \mathrm{~h}$. To quantify the apoptotic cells, terminal deoxynucleotidyl transferase-mediated nick end labeling (TUNEL)-positive cells were counted using confocal microscopy (Olympus, Tokyo, Japan).

In addition, at the molecular level, we also detected other anti-apoptotic molecules, such as survivin, and Bcl-2 protein expression by western blotting as an additional indicator of apoptosis.
Caspase activity. The activities of caspase-3 and -9 were measured using Caspase Colorimetric Protease Assay kits (Millipore Corp., Billerica, MA, USA) according to the manufacturer's instructions. In brief, A549 cells were treated with the indicated plasmid for $24 \mathrm{~h}$, and washed twice with ice-cold PBS (pH 7.2) and harvested by centrifugation. The cell pellets were then lysed in $150 \mu \mathrm{l}$ buffer provided in the kit (Millipore). An aliquot of lysates $(80 \mu \mathrm{l})$ was incubated with $10 \mu \mathrm{l}$ substrate of each caspase at $37^{\circ} \mathrm{C}$ for $2 \mathrm{~h}$. Samples were analyzed at $405 \mathrm{~nm}$ in a microplate reader (Thermo Fisher Scientific Inc., Waltham, MA, USA). The relative caspase activity of the control group was taken as 100 . Each assay was conducted in triplicate.

Wound healing assay. To assess the effect of XIAP-shRNA in combination with $\mathrm{CXB}$ on cell migration, a wound-healing assay was performed. In brief, $2 \times 10^{5}$ A549 cells were plated in 6-well plates in RMP-1640 medium containing 10\% FBS. After $24 \mathrm{~h}$, a scratch was made through the confluent cell monolayer, and the cells were treated with the indicated agent, respectively, in $3 \mathrm{ml}$ of complete medium. After a 48-h treatment, cells were stained with hematoxylin and eosin (H\&E). Cells invading the wound line were observed under an inverted phase-contrast microscope (Leica DMR, Germany). Triplicates were repeated in all experiments.

Matrigel invasion assay. The migration capacity of NSCLC cells was determined in vitro using Transwell chambers (Corning, Tewksbury, MA, USA) in which the two chambers were separated by a Matrigel-coated polycarbonate membrane $(8-\mu \mathrm{m}$ pore size). Cells were treated with CXB, XIAP-shRNA, or the combination of the two agents for $24 \mathrm{~h}$ in FBS-free RPMI-1640 medium. The lower chamber was then filled with $500 \mathrm{ml}$ of RPMI-1640 medium supplemented with $10 \%$ FBS and $5 \times 10^{5}$ cells $/ \mathrm{ml}$ were placed in the upper chamber in a total volume of $200 \mathrm{ml}$. The Transwell chamber was then assembled, and the cells were incubated for $24 \mathrm{~h}$ at $37^{\circ} \mathrm{C}$ in a $5 \% \mathrm{CO}_{2}$ environment. After a 24-h incubation, cells invading the bottom surface of the filter were fixed and stained with $0.1 \%$ crystal violet in $20 \%$ methanol. Invasiveness was determined by counting the penetrating cells under a Nikon phase-contrast microscope and counted in $>10$ fields of view at x200 magnification. The invasion of cells without any treatment was determined as $100 \%$. Each cell migration experiment was repeated three times.

In addition, we also detected MMP-9 and MMP-2 protein expression by western blotting as an additional indicator of invasion and migration.

Measurement of prostaglandin-E2 (PGE2) production and VEGF expression. Prostaglandin-E2 (PGE2) production was determined by competitive enzyme-linked immunosorbent assay (ELISA). In brief, A549 cells were treated with XIAP-shRNA, CXB, alone or both for $48 \mathrm{~h}$ in 12-well plates, and then the culture media were centrifuged to remove the cell debris. Cell-free culture media were collected at the indicated times. Then PGE2 levels were measured by using Human Prostaglandin E2 ELISA kits (Kemin, Shanghai, China) according to the manufacturer's instructions.

A549 cells were treated with XIAP-shRNA, CXB or both for $48 \mathrm{~h}$ in 24 -well plates, and then the culture media were 
centrifuged to remove cell debris. Cell-free culture media were collected at the indicated times. The VEGF level in the cell supernatant was determined by using the Human VEGF ELISA kit (Yanyu, Shanghai, China) according to the manufacturer's instructions.

Tumor xenograft experiments. Specific pathogen-free (SPF) nude mice $(\mathrm{BALB} / \mathrm{c})$ used in the experiments were provided by the Center for Experimental Animals at Jilin University (Jilin, China). All experiments involving animals were performed in accordance with institutional guidelines, following a protocol approved by the Ethics Committees of the Disease Model Research Center, The First Hospital of Jilin University. Forty female BALB mice (aged 6-7 weeks) were maintained under specific pathogen-free conditions and provided with food and water ad libitum.

Stable A549/XIAP-shRNA clones were harvested, and a tumorigenic dose of $2.5 \times 10^{6}$ cells was injected intraperitoneally in 20 mice. The remaining 20 mice were injected with untreated A549 cells as the control group. When progression was evident in 28 days, 10 mice from each group were randomly selected and were treated with CXB ( $4.56 \mathrm{mg} / \mathrm{kg}$ body weight) intraperitoneally on alternative days for 2 weeks, respectively. Tumor growth was recorded twice a week, starting with the first day of treatment. The mice were euthanized 14 days after CXB treatment, and the subcutaneous tumors were isolated, weighed and volume measured. Parts of each tumor tissue were wax embedded for H\&E staining to study cell apoptosis in vivo by TUNEL assay.

In situ TUNEL assay. TUNEL analysis for apoptotic cells in the tumor tissues was performed with the Dead End ${ }^{\mathrm{TM}}$ Fluorometric TUNEL System (Promega, Madison, WI, USA) according to the manufacturer's protocol.

Statistical analysis. All data are expressed as mean values \pm SD. Statistical analysis between two samples was performed using the Student's t-test. Statistical comparison of more than two groups was performed using one-way ANOVA followed by a Tukey post hoc test. The Graphpad Prism 5.0 software (GraphPad Software, San Diego, CA, USA) was used for statistical analyses. $\mathrm{P}<0.05$ was considered to indicate a statistically significant difference.

\section{Results}

Specific downregulation of XIAP expression by XIAP-shRNA. The mRNA and protein expression levels of the XIAP gene, inhibited by specific shRNAs in the NSCLC cells, were analyzed by real-time quantitive RT-PCR and western blotting. As shown in Fig. 1A, the level of XIAP mRNA in the XIAP-shRNA group was significantly decreased compared with these levels in the control group and the NC-shRNA group, respectively $(\mathrm{P}<0.05)$; no significant difference was noted between the NC-shRNA group and the control group. Additionally, at the protein expression level, no significant inhibition of XIAP protein expression was found in the NC-shRNA group and control group ( $\mathrm{P}>0.05)$, while the band density decreased markedly in the XIAP-shRNA group as compared with the NC-shRNA and control groups $(\mathrm{P}<0.05$,
A

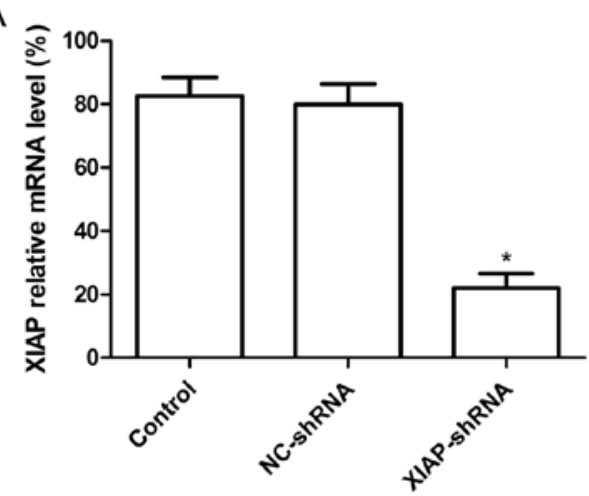

B

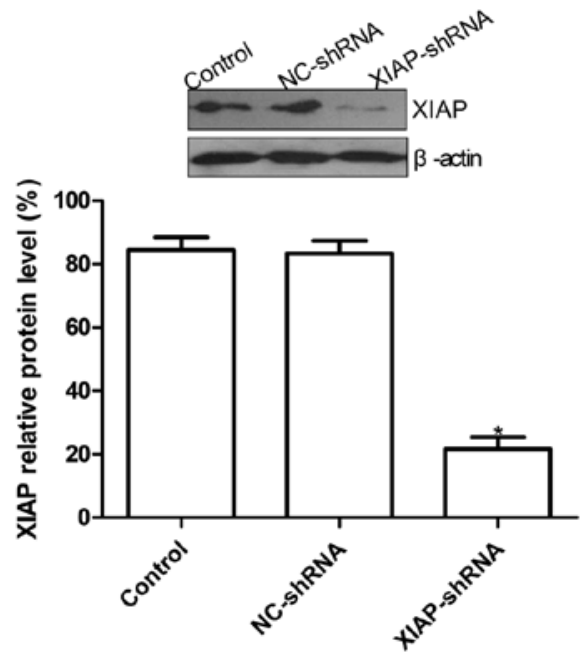

Figure 1. Detection of XIAP mRNA and protein expression by RT-qPCR and western blot assay, respectively. (A) Quantitative RT-PCR of XIAP mRNA expression in A549 cells $48 \mathrm{~h}$ after transfection with XIAP-shRNA. Data were collected from three independent experiments. (B) Western blot analysis of XIAP protein levels in A549 cells $48 \mathrm{~h}$ after transfection with XIAP-shRNA ( $\beta$-actin was included as an internal control). Data were collected from three independent experiments; ${ }^{*} \mathrm{P}<0.05$ vs. control group.

Fig. 1B). The above results indicate that the expression of the XIAP gene in the A549 cells was specifically and effectively downregulated by the shRNA targeting XIAP.

Effects of CXB and XIAP-shRNA alone or in combination on A549 cell proliferation and apoptosis. To evaluate the effect of XIAP-shRNA and CXB alone and in combination on the cell proliferation of NSCLC cells in vitro, MTT assay was performed in A549 cells $48 \mathrm{~h}$ after treatment with XIAPshRNA and CXB alone or the combination. It was found that the inhibitory rates of the cells treated with XIAP-shRNA and $\mathrm{CXB}$ alone or the combination were higher than those of the control group and NC-shRNA group $(\mathrm{P}<0.05$, Fig. $2 \mathrm{~A})$. No significant difference was noted between the control group and the NC-shRNA group ( $\mathrm{P}>0.05$, Fig. $2 \mathrm{~A}$ ). In addition, the inhibitory rate of the combination group was higher than the rates in the single treatment groups $(\mathrm{P}<0.05$, Fig. $2 \mathrm{~A})$.

The effects of XIAP-shRNA and CXB alone or the combination on A549 cell apoptosis were then analyzed by TUNEL assay. Compared with control group and NC-shRNA group, XIAP-shRNA and CXB alone or the combination groups had 
A

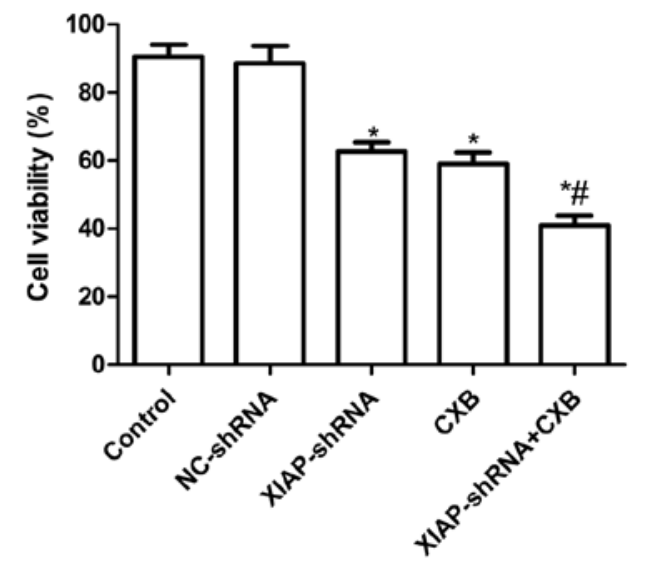

B

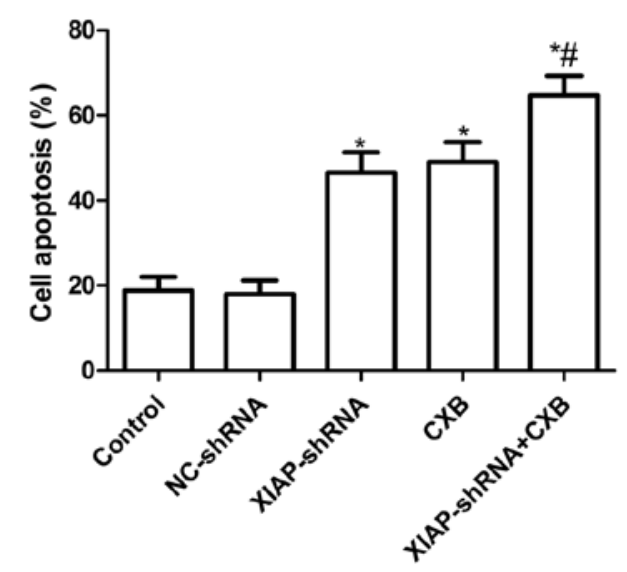

Figure 2. Effect of CXB and XIAP-shRNA alone and in combination on cell proliferation and apoptosis. (A) Cell proliferation and (B) apoptosis of A549 cells were determined after treatment with $\mathrm{CXB}$ and XIAP-shRNA alone or in combination. $\mathrm{P}<0.05$ vs. control; ${ }^{*} \mathrm{P}<0.05$ vs. CXB alone.

A

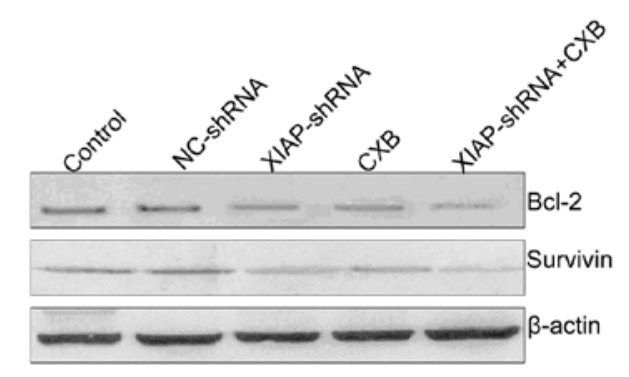

C

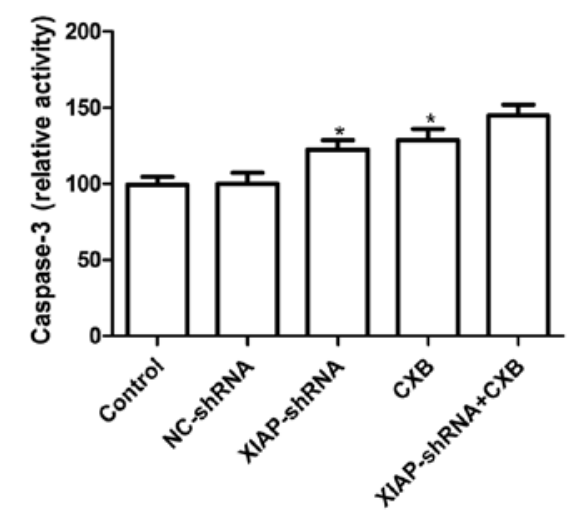

B
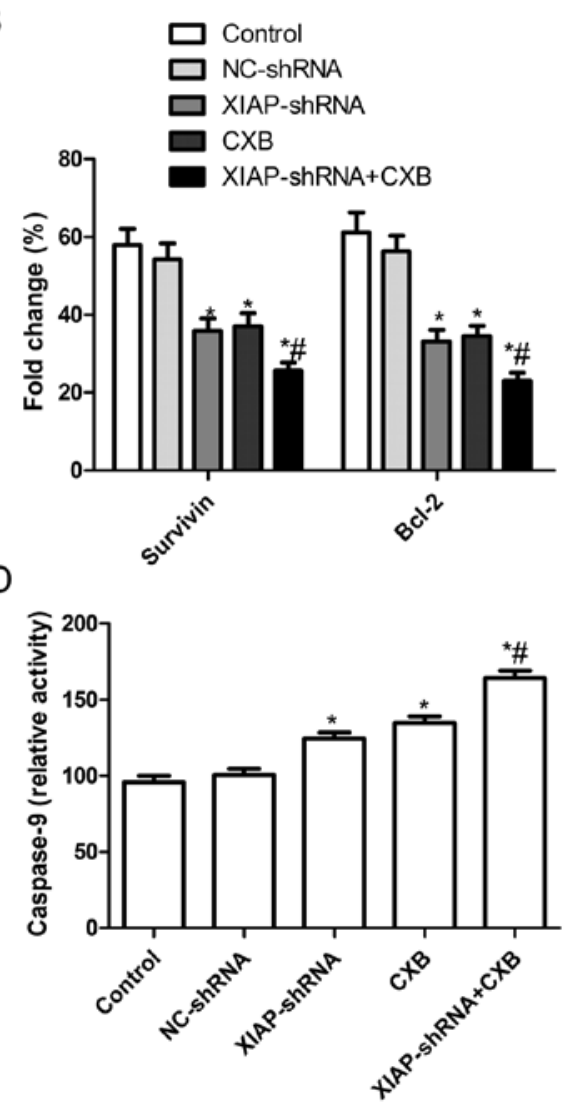

Figure 3. Effects of CXB and XIAP-shRNA alone or in combination on expression of anti-apoptotic molecules and caspase activity in A549 cells. (A) Survivin and Bcl-2 expression levels were determined by western blotting ( $\beta$-actin was included as an internal control). (B) Relative quantification of survivin and Bcl-2 protein by densitometric analysis. (C) Caspase-3 and (D) caspase- 9 activity were measured by ELISA; ${ }^{*} \mathrm{P}<0.05$ vs. control; ${ }^{\#} \mathrm{P}<0.05$ vs. CXB alone.

significantly induced cell apoptosis $(\mathrm{P}<0.05$, Fig. $2 \mathrm{~B})$. The combination group had a greater percentage of cell apoptosis than either drug alone treated group ( $\mathrm{P}<0.05$, Fig. $2 \mathrm{~B})$.

Effects of CXB and XIAP-shRNA alone or in combination on the expression of anti-apoptotic molecules and caspase activity in A549 cells. To explore the possible mechanism of induction of cell apoptosis by CXB and XIAP-shRNA alone or the combination in A549 cells, expression patterns of survivin, and Bcl-2 were determined by western blotting. The results showed that XIAP-shRNA and CXB alone or the combination significantly decreased the expression of apoptosis inhibiting genes survivin and Bcl-2 in the A549 cells compared to levels in the control group and the NC-shRNA group $(\mathrm{P}<0.05$, Fig. $3 \mathrm{~A}$ and $\mathrm{B}$ ). Compared to the single treatment groups, the combination treatment led to markedly decreased survivin and 
A
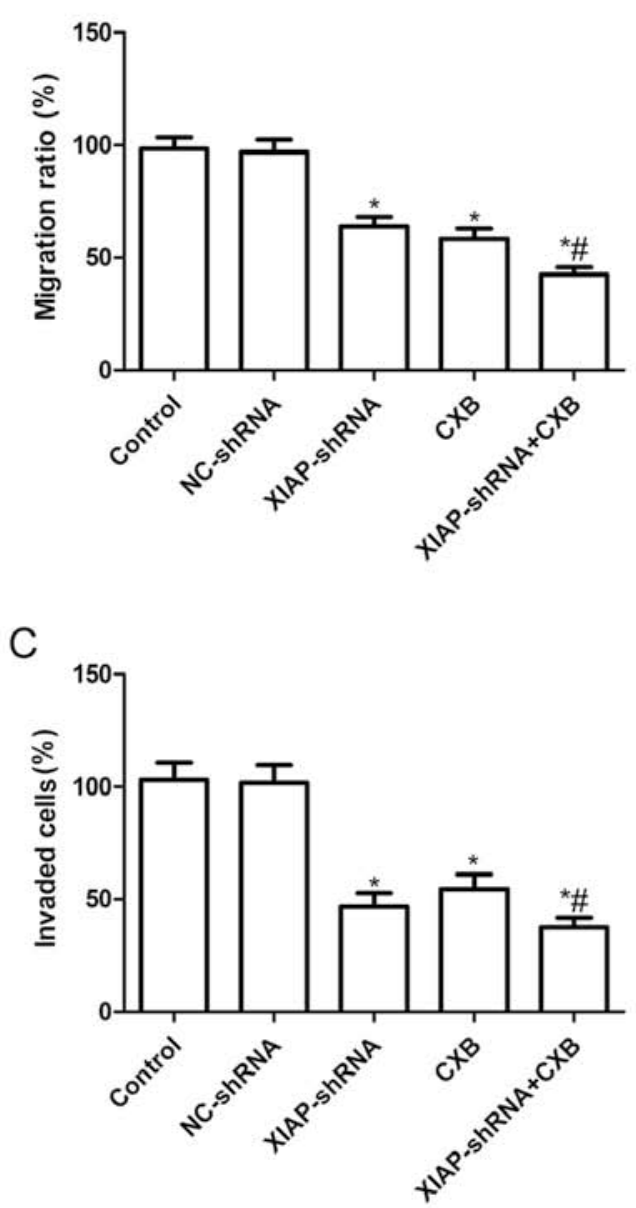

B

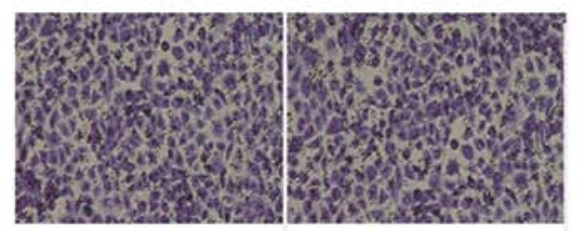

Control

NC-shRNA

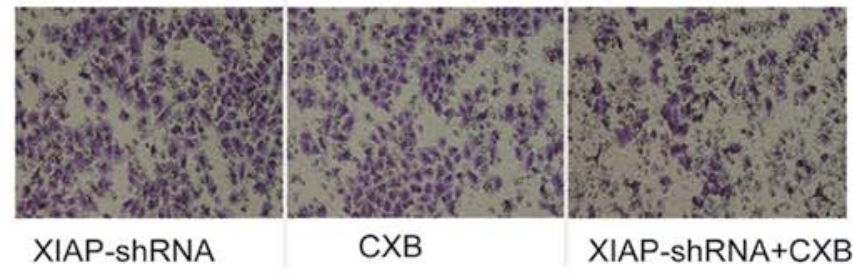

D

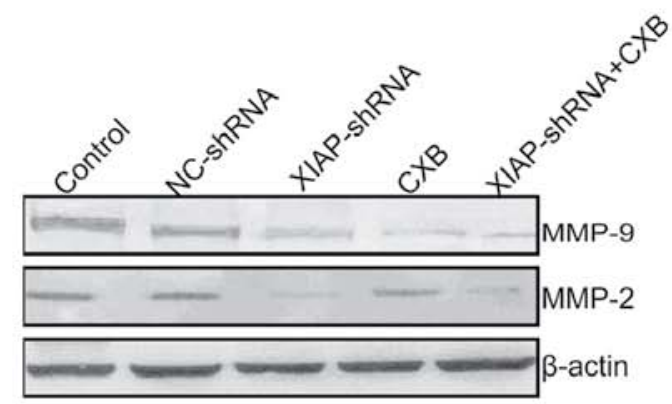

Figure 4. Effect of CXB and XIAP-shRNA alone and in combination on cell migration and invasion in A549 cells. (A) Cell migration was determined $48 \mathrm{~h}$ after treatment with CXB and XIAP-shRNA alone or in combination. (B) Cell invasion was determined by Matrigel after treatment with CXB and XIAP-shRNA alone or in combination. A549 cells under the chamber were stained by crystal violet. (C) Histogram of the percentage of invaded cells in each group, normalized to the control cells. (D) Expression of MMP-2 and MMP-9 in A549 cells was determined by western blotting. Data are expressed as the mean values $\pm \mathrm{SD}$. ${ }^{*} \mathrm{P}<0.05$ vs. control; ${ }^{*} \mathrm{P}<0.05$ vs. $\mathrm{CXB}$ alone.

Bcl-2 protein expression ( $\mathrm{P}<0.05$, Fig. $3 \mathrm{~A}$ and $\mathrm{B})$. Additionally, caspase-3 and caspase- 9 activity were then determined by ELISA. The results showed that XIAP-shRNA and CXB alone or the combination significantly increased caspase-3 and caspase- 9 activity compared to the control group and the NC-shRNA group ( $\mathrm{P}<0.05$, Fig. $3 \mathrm{C}$ and $\mathrm{D})$, and the combination group resulted in a greater added increase compared to the single drug treatment groups.

Effects of CXB and XIAP-shRNA alone or the combination on cell migration and invasion. To ascertain the inhibitory effect of XIAP-shRNA and CXB alone or the combination on NSCLC cell motility in vitro, a wound-healing assay was performed. As shown in Fig. 4A, cells in the XIAP-shRNA and $\mathrm{CXB}$ alone and the combination group migrated significantly less than those of the control group and the NC-shRNA group when A549 cells were treated with XIAP-shRNA and CXB alone or combination treatment for $48 \mathrm{~h}(\mathrm{P}<0.05)$. In addition, compared to the single drug treatments, the migratory ability was significantly reduced in the CXB and XIAP-shRNA combination group ( $\mathrm{P}<0.05$, Fig. 4A).
The invasion potential of treated cells was evaluated by a Transwell assay. As shown in Fig. 4B, the result of the cell invasion assay showed that there was no significant difference in the number of cells that passed through the simulated basement membrane between the control group and the NC-shRNA group. However, the number of cells that passed through the simulated basement membrane in the XIAP-shRNA and CXB alone or the combination group was significantly reduced when compared with the control group and NC-shRNA group. Moreover, the invasiveness of A549 cells was further attenuated when the cells received the combination treatment as compared with the single treatment groups.

To determine the potential mechanism of the inhibition of cell invasion and migration by the combination treatment of CXB and XIAP-shRNA in vitro, levels of MMP-2 and MMP-9 protein expression were determined by western blotting. Western blot analysis displayed a significant decrease in MMP-2 and MMP-9 proteins in the XIAP-shRNA and CXB alone and in the combination group when compared to these levels in the control group and the NC-shRNA group $(\mathrm{P}<0.05$, Fig. $4 \mathrm{C}$ and D). As compared to the single treatment groups, 
A

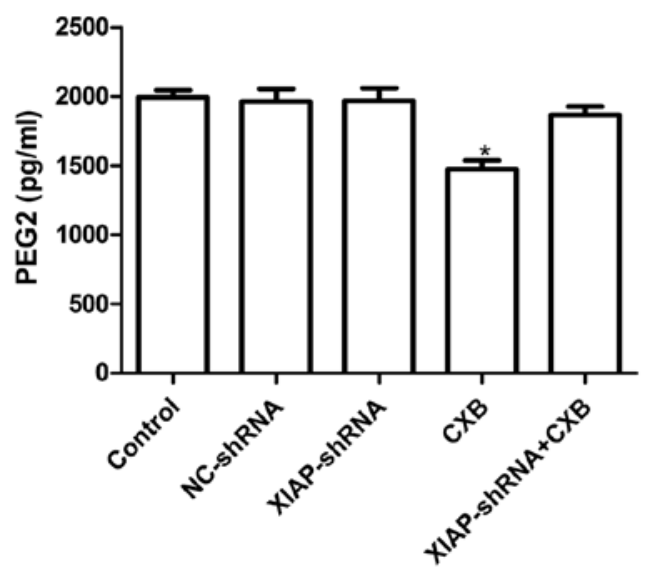

B

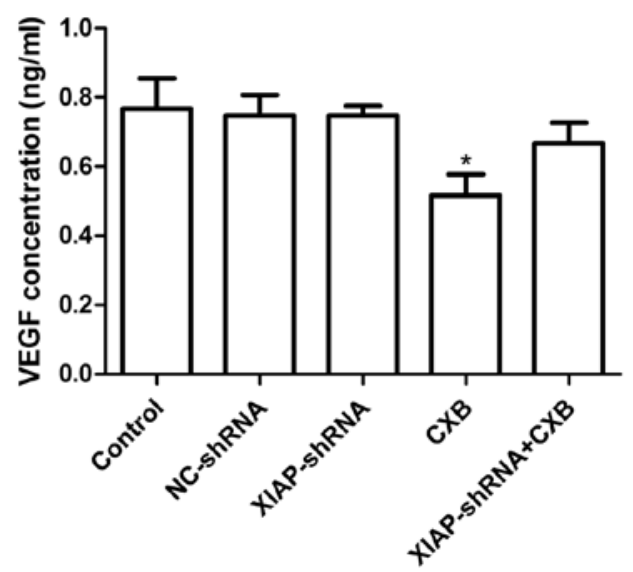

Figure 5. Effect of CXB and XIAP-shRNA alone or in combination on prostaglandin-E2 (PGE2) production and VEGF expression in A549 cells. (A) PGE2 production and (B) VEGF expression were measured by ELISA. Data are expressed as mean values $\pm \mathrm{SD}$. ${ }^{*} \mathrm{P}<0.05$ vs. control; ${ }^{\#} \mathrm{P}<0.05$ vs. $\mathrm{CXB}$ alone.

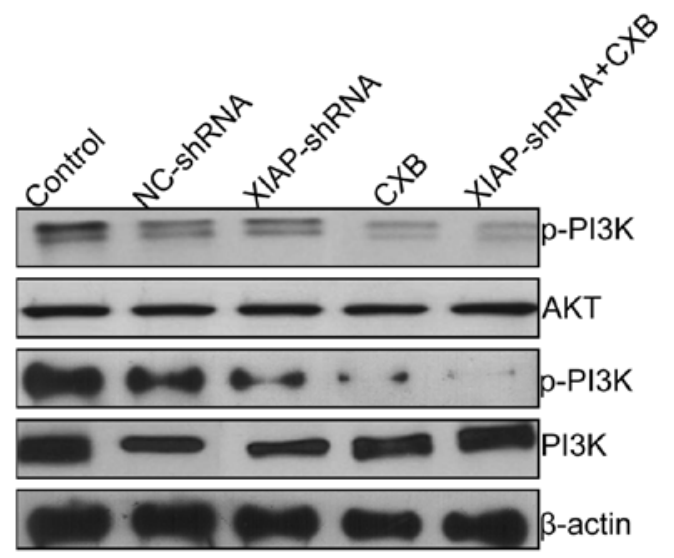

Figure 6. Effect of CXB and XIAP-shRNA alone or in combination on the PI3K/AKT signaling pathway in A549 cells. Phosphorylation/activation pattern of PI3K and Akt were measured by western blotting $24 \mathrm{~h}$ after treatment with CXB and XIAP-shRNA alone or in combination in the A549 cell line. Blots were reprobed for $\beta$-actin to normalize each lane for protein content.

the combination group exhibited obviously decreased MMP-2 and MMP-9 protein expression ( $\mathrm{P}<0.05$, Fig. 4C and D).

Effects of CXB and XIAP-shRNA alone or the combination on $P G E 2$ production and the VEGF level. To examine the effects of XIAP-shRNA and CXB alone and in combination on PGE2 production in A549 cells, ELISA was performed. As shown in Fig. 5A, CXB inhibited significant $\mathrm{PGE} 2$ production $(\mathrm{P}<0.05)$; however, $\mathrm{CXB}$ in combination with XIAP-shRNA or XIAP-shRNA alone did not inhibit PGE2 production. We also determined the VEGF level by ELISA. As shown in Fig. 5B, ELISA analysis revealed that VEGF excretion in the supernatant from the CXB group was significantly decreased compared to the other groups $(\mathrm{P}<0.05)$. $\mathrm{CXB}$ in combination with XIAP-shRNA or XIAP-shRNA alone did not inhibit VEGF protein expression.

Effects of CXB and XIAP-shRNA alone or the combination on the PI3K/Akt pathway. It has been demonstrated that celecoxib (CXB) is involved in Akt phosphorylation inhibition and regulates cell apoptosis (18). We aimed to ascertain whether the mediation of this signaling pathway could be enhanced by CXB in combination with XIAP-shRNA. Measurement of the phosphorylation/activation pattern of PI3K and Akt was performed by western blotting. Our results showed that CXB and XIAP-shRNA alone or the combination resulted in a marked reduction in phosphorylated $\mathrm{PI} 3 \mathrm{~K}$ and Akt relative to the control group and NC-shRNA group, without altering the total protein levels of PI3K or Akt in each group (Fig. 6). Compared to the CXB and XIAP-shRNA alone treatment groups, the combination group exhibited obviously decreased phosphorylation of PI3K and Akt protein expression, demonstrating a synergistic effect.

Effects of CXB and XIAP-shRNA alone or the combination on tumor growth in vivo. Finally, we assessed the in vivo therapeutic efficacy of XIAP-shRNA and CXB alone or the combination on female BALB mice bearing A549 cell tumors. In the XIAP-shRNA treatment group, the tumor size and weight were reduced to 63.61 and $53.23 \%$; in the CXB treated group, the tumor size and weight were reduced to 56.13 and $37.08 \%$; and in the combination group, tumor size and weight were reduced to 36.12 and $17.79 \%$. These results suggest that the most prominent tumor decrease occurred in the combination group, demonstrating the synergistic effect in vivo $(\mathrm{P}<0.05$, Fig. $7 \mathrm{~A}-\mathrm{C})$.

In addition, we also determined the synergistic effects on tumor tissue cell apoptosis in vivo by TUNEL. The results showed that XIAP-shRNA and CXB alone or the combination significantly induced cell apoptosis compared to the control group and the NC-shRNA group ( $\mathrm{P}<0.05$, Fig. 7D). The combination of XIAP-shRNA and CXB greatly induced tumor cell apoptosis in vivo compared to the single drug treatment groups $(\mathrm{P}<0.05$, Fig. 7D).

\section{Discussion}

Non-small cell lung cancer is one of the leading causes of cancer-related death in many countries (27). Despite the fact that advances in diagnostic and therapeutic methodolo- 
A

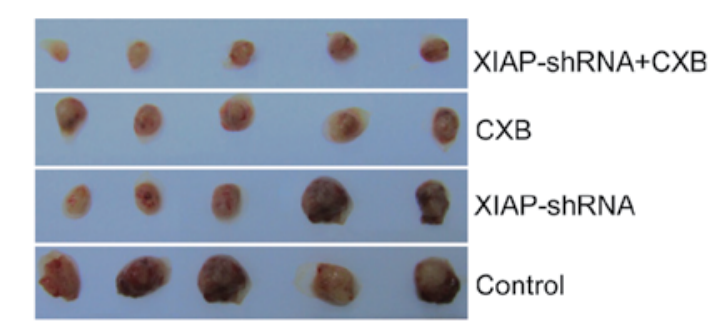

C

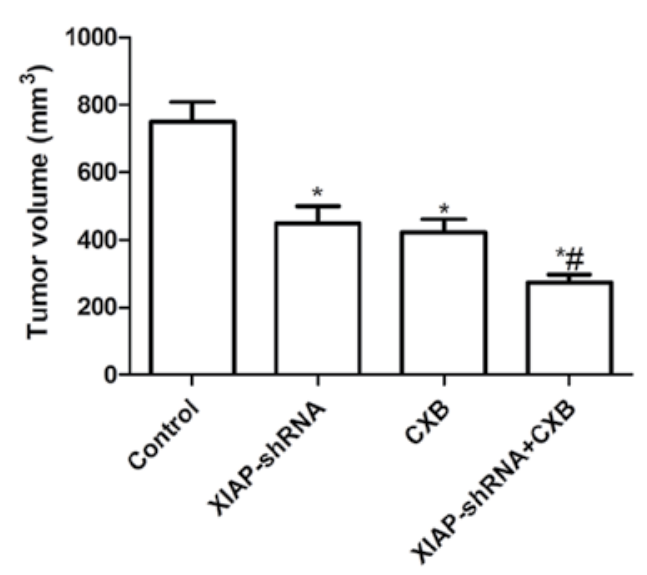

$\mathrm{B}$

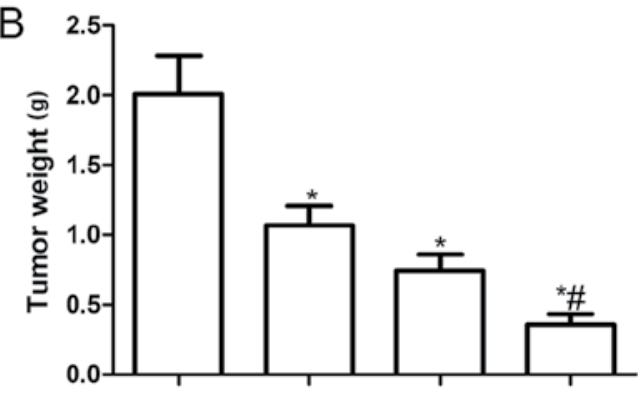

D

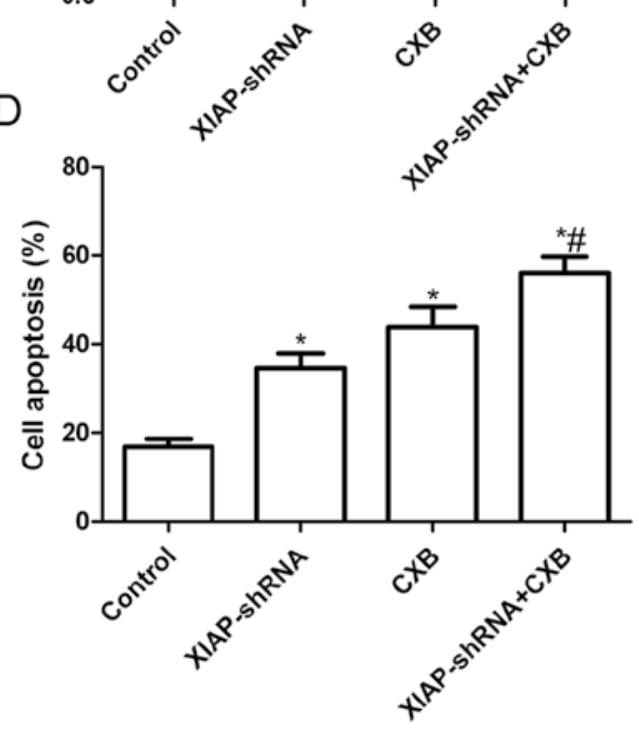

Figure 7. Antitumor activity of CXB and XIAP-shRNA alone or in combination in BALB/c mice bearing A549 cell tumors. (A) Images of the tumor tissues from the different groups. (B) Tumor weight in each group. (C) Tumor volume in each group. (D) TUNEL assay of cell apoptosis in vivo. Data are expressed as mean values $\pm \mathrm{SD}$. ${ }^{*} \mathrm{P}<0.05$ vs. control; ${ }^{\#} \mathrm{P}<0.05$ vs. $\mathrm{CXB}$ alone.

gies have been exploited to improve the survival of patients with NSCLC, a satisfactory level has not been achieved as the majority of patients present at an advanced unresectable stage and their tumors exhibit an inherent resistance to chemotherapy and radiotherapy. Therefore, the development of new treatment strategies with high efficacy and low toxicity is required for NSCLC (28).

It is well known that combination treatments achieve a better curative effect when compared to single drug treatments. Combination treatment mainly depends on the synergism between different therapeutic agents with different mechanisms of action to increase efficacy, while maintaining a favorable side effect and toxicity profile. One example of a drug that is commonly used as a component of combination therapies is CXB. It is one of the most widely used chemotherapeutic agents for the treatment of lung cancer (21). Although CXB induces substantial antitumor activity and cytotoxicity in various cancer types (29-31), it also results in the development of resistance through altered bioavailability or inactivation and nonspecific cytotoxicity (32). Several studies have demonstrated that the response rate with the combination of CXB and gefitinib for advanced NSCLC patients was similar to that with gefitinib alone $(33,34)$. Recently, Koch et al found that patients with advanced NSCLC at stage IIIB-IV who received CXB plus chemotherapy also failed to demonstrate a survival benefit for CXB (35). In addition, CXB causes various side effects at the therapeutic dosage including cardiovascular thrombosis, congestive heart failure, gastrointestinal ulceration, renal or hepatic injury, and platelet aggregation (22). Therefore, by reducing the dosage of CXB the cytotoxic effects may be achieved by using combination therapy.

One of the major factors responsible for chemoresistance is the evolution of tumor cells towards a phenotype that is resistant to apoptotic cell killing. Cells resist apoptosis by a key molecular mechanism, the overexpression of inhibitor of apoptosis protein (IAP) family of proteins. A key member of this family is XIAP. It has been showed that XIAP overexpression is correlated with resistance to apoptosis through stimulation of both the intrinsic (mitochondrial-directed) and extrinsic (death receptor-directed) pathways $(36,37)$. Several studies have shown that downregulation of XIAP with siRNA restores chemosensitivity in various tumor cell lines (9-14). Pan et al showed that the combination of XIAP-shRNA and TRAIL resulted in significant reduction in XIAP expression and potent antitumor activity both in HCC cells and in an animal tumor model (38). Therefore, in this study, we selected XIAP-shRNA in combination with a low dose of CXB for NSCLC. Our results showed that XIAP-shRNA combined with a low dose of CXB inhibited NSCLC tumor growth in vitro and in an animal model.

Although the combination therapy improves the response rate and time to progression compared with sequential monotherapy, it causes increased toxicity. Consequently, in 
cases of advanced cancer, emerging guidelines recommend sequential monotherapy to improve quality of life. An alternative approach that could overcome nonspecific toxicity while retaining therapeutic efficacy, involves the combination of chemotherapy with targeted therapy by RNAi technology. Recent research shows that combined therapy with an shRNA targeting gene in combination with an anticancer drug could decrease toxicity and chemoresistance, and achieve better antitumor activity (39). For example, Ghosh et al showed that sequence-dependent combination treatment using lowdose doxorubicin and an anti-survivin agent was efficacious against both breast and pancreatic cancer (39). Du et al showed that treatment with a lentivirus carrying the COX-2 gene in combination with tamoxifen in breast cancer cells significantly suppressed tumor growth in vitro and in vivo (40). Xue et al found that the combination of sur-shRNA and emodin was effective for the treatment of ovarian cancer compared to sur-shRNA and emodin alone treatment (41). Consistent with these results, our results demonstrated that XIAP-shRNA in combination with CXB treatment in NSCLC significantly suppressed the proliferation, migration and invasion, and induced cell apoptosis in vitro, and inhibited tumor growth in a mouse model.

The PI3K/Akt pathway plays an important role in lung cancer pathogenesis, the upregulation of which is associated with a more aggressive clinical phenotype and worse clinical outcome for endocrine-treated patients (42-44). It has been showed that CXB inhibits the expression of phosphorylated-AKT and regulates cell apoptosis (18). In the present study, our results showed that CXB and XIAP-shRNA alone or the combination resulted in a marked reduction in phosphorylated PI3K and Akt relative to the control group and NC-shRNA group, without altering the total protein levels of PI3K or Akt in each group. XIAP-shRNA in combination with CXB obviously decreased phosphorylated PI3K and Akt protein expression compared to the CXB and XIAP-shRNA alone treatment groups, demonstrating a synergistic effect. In addition, our study showed that CXB inhibited PGE2 production and VEGF expression, which was consistent with a previous study (42). However, CXB in combination with XIAP-shRNA or XIAP-shRNA alone did not inhibit PGE2 production and VEGF expression, which suggests that $\mathrm{CXB}$ in combination with XIAP-shRNA did not have a synergistic effect on PGE2 production and VEGF expression in A549 cells. These findings imply that XIAP-shRNA in combination with $\mathrm{CXB}$ has a synergistic effect on the inhibition of tumor growth of NSCLC in vitro and in vivo, at least in part, through affecting the PI3K/AKT signaling pathway.

In conclusion, our in vitro studies demonstrated that when plasmid XIAP-shRNA and CXB are used in combination, they provide an effective therapeutic approach for NSCLC. This combination significantly suppressed the proliferation, migration and invasion, and induced cell apoptosis when compared to treatment with XIAP-shRNA and CXB alone. Additionally, further studies with in vivo mouse models also confirmed that this combination suppressed the tumor growth of NSCLC. These findings suggest that the combination of XIAP-shRNA and $\mathrm{CXB}$ is a promising drug candidate for the treatment of NSCLC.

\section{Acknowledgements}

This research was supported by the Science and Technology Research and Innovation Team Fund of Jilin Province (JL2013038).

\section{References}

1. Jemal A, Bray F, Center MM, Ferlay J, Ward E and Forman D: Global cancer statistics. CA Cancer J Clin 61: 69-90, 2011.

2. Reungwetwattana T, Weroha SJ and Molina JR: Oncogenic pathways, molecularly targeted therapies, and highlighted clinical trials in non-small-cell lung cancer (NSCLC). Clin Lung Cancer 13: 252-266, 2012.

3. Zhang H, Li Z and Wang K: Combining sorafenib with celecoxib synergistically inhibits tumor growth of non-small cell lung cancer cells in vitro and in vivo. Oncol Rep 31: 1954-1960, 2014.

4. Hunter AM, LaCasse EC and Korneluk RG: The inhibitors of apoptosis (IAPs) as cancer targets. Apoptosis 12: 1543-1568, 2007.

5. Danson S, Dean E, Dive C and Ranson M: IAPs as a target for anticancer therapy. Curr Cancer Drug Targets 7: 785-794, 2007.

6. Devi GR: XIAP as target for therapeutic apoptosis in prostate cancer. Drug News Perspect 17: 127-134, 2004.

7. Stennicke HR, Ryan CA and Salvesen GS: Reprieval from execution: the molecular basis of caspase inhibition. Trends Biochem Sci 27: 94-101, 2002.

8. Holcik M, Gibson H and Korneluk RG: XIAP: apoptotic brake and promising therapeutic target. Apoptosis 6: 253-261, 2001.

9. Ma JJ, Chen BL and Xin XY: XIAP gene downregulation by small interfering RNA inhibits proliferation, induces apoptosis, and reverses the cisplatin resistance of ovarian carcinoma. Eur J Obstet Gynecol Reprod Biol 146: 222-226, 2009.

10. Yamaguchi Y, Shiraki K, Fuke H, et al: Targeting of X-linked inhibitor of apoptosis protein or survivin by short interfering RNAs sensitize hepatoma cells to TNF-related apoptosisinducing ligand- and chemotherapeutic agent-induced cell death. Oncol Rep 14: 1311-1316, 2005.

11. Jiang C, Yi XP, Shen H and Li YX: Targeting X-linked inhibitor of apoptosis protein inhibits pancreatic cancer cell growth through p-Akt depletion. World J Gastroenterol 18: 2956-2965, 2012.

12. Kwatra SG: Targeting X-linked inhibitor of apoptosis protein for melanoma therapy: the need for more homogeneous samples and the importance of cell lines. J Invest Dermatol 131: 797, 2011.

13. Hiscutt EL, Hill DS, Martin S, et al: Targeting X-linked inhibitor of apoptosis protein to increase the efficacy of endoplasmic reticulum stress-induced apoptosis for melanoma therapy. J Invest Dermatol 130: 2250-2258, 2010.

14. Mastrangelo E, Cossu F, Milani M, et al: Targeting the $\mathrm{X}$-linked inhibitor of apoptosis protein through 4-substituted azabicyclo[5.3.0]alkane smac mimetics. Structure, activity, and recognition principles. J Mol Biol 384: 673-689, 2008.

15. Harlin H, Reffey SB, Duckett CS, Lindsten T and Thompson CB: Characterization of XIAP-deficient mice. Mol Cell Biol 21: 3604-3608, 2001

16. Wang ZL, Fan ZQ, Jiang HD and Qu JM: Selective Cox-2 inhibitor celecoxib induces epithelial-mesenchymal transition in human lung cancer cells via activating MEK-ERK signaling. Carcinogenesis 34: 638-646, 2013.

17. Dhawan D, Jeffreys AB, Zheng R, Stewart JC and Knapp DW: Cyclooxygenase-2 dependent and independent antitumor effects induced by celecoxib in urinary bladder cancer cells. Mol Cancer Ther 7: 897-904, 2008.

18. Kulp SK, Yang YT, Hung CC, et al: 3-phosphoinositidedependent-protein kinase-1/Akt signaling represents a major cyclooxygenase-2-independent target for celecoxib in prostate cancer cells. Cancer Res 64: 1444-1451, 2004.

19. Chen ST, Thomas S, Gaffney KJ, Louie SG, Petasis NA and Schönthal AH: Cytotoxic effects of celecoxib on Raji lymphoma cells correlate with aggravated endoplasmic reticulum stress but not with inhibition of cyclooxygenase-2. Leuk Res 34: 250-253, 2010.

20. Jendrossek V, Handrick R and Belka C: Celecoxib activates a novel mitochondrial apoptosis signaling pathway. FASEB J 17: 1547-1549, 2003. 
21. Liu X, Yue P, Zhou Z, Khuri FR and Sun SY: Death receptor regulation and celecoxib-induced apoptosis in human lung cancer cells. J Natl Cancer Inst 96: 1769-1780, 2004.

22. Menter DG, Schilsky RL and DuBois RN: Cyclooxygenase-2 and cancer treatment: understanding the risk should be worth the reward. Clin Cancer Res 16: 1384-1390, 2010.

23. Jeon YW and Suh YJ: Synergistic apoptotic effect of celecoxib and luteolin on breast cancer cells. Oncol Rep 29: 819-825, 2013.

24. Cervello M, Bachvarov D, Lampiasi N, et al: Novel combination of sorafenib and celecoxib provides synergistic anti-proliferative and pro-apoptotic effects in human liver cancer cells. PLoS One 8: e65569, 2013.

25. Morisaki T,Umebayashi M,Kiyota A, et al: Combining celecoxib with sorafenib synergistically inhibits hepatocellular carcinoma cells in vitro. Anticancer Res 33: 1387-1395, 2013.

26. Huang KH, Kuo KL, Chen SC, et al: Down-regulation of glucose regulated protein (GRP) 78 potentiates cytotoxic effect of celecoxib in human urothelial carcinoma cells. PLoS One 7 : e33615, 2012.

27. Jemal A, Siegel R, Ward E, Hao Y, Xu J and Thun MJ: Cancer statistics, 2009. CA Cancer J Clin 59: 225-249, 2009.

28. Mendez M, Custodio A and Provencio M: New molecular targeted therapies for advanced non-small-cell lung cancer. J Thorac Dis 3: 30-56, 2011.

29. Dhawan D, Craig BA, Cheng L, et al: Effects of short-term celecoxib treatment in patients with invasive transitional cell carcinoma of the urinary bladder. Mol Cancer Ther 9: 1371-1377, 2010.

30. Liu X, Yue P, Schönthal AH, Khuri FR and Sun SY: Cellular FLICE-inhibitory protein down-regulation contributes to celecoxib-induced apoptosis in human lung cancer cells. Cancer Res 66: 11115-11119, 2006.

31. Huang S and Sinicrope FA: Celecoxib-induced apoptosis is enhanced by ABT-737 and by inhibition of autophagy in human colorectal cancer cells. Autophagy 6: 256-269, 2010

32. Davies NM, McLachlan AJ, Day RO and Williams KM: Clinical pharmacokinetics and pharmacodynamics of celecoxib: a selective cyclo-oxygenase-2 inhibitor. Clin Pharmacokinet 38 225-242, 2000

33. Gadgeel SM, Ruckdeschel JC, Heath EI, Heilbrun LK, Venkatramanamoorthy R and Wozniak A: Phase II study of gefitinib, an epidermal growth factor receptor tyrosine kinase inhibitor (EGFR-TKI), and celecoxib, a cyclooxygenase-2 (COX-2) inhibitor, in patients with platinum refractory non-small cell lung cancer (NSCLC). J Thorac Oncol 2: 299-305, 2007.
34. Agarwala A, Fisher W, Bruetman D, et al: Gefitinib plus celecoxib in chemotherapy-naive patients with stage IIIB/IV non-small cell lung cancer: a phase II study from the Hoosier Oncology Group. J Thorac Oncol 3: 374-379, 2008.

35. Koch A, Bergman B, Holmberg E, et al: Effect of celecoxib on survival in patients with advanced non-small cell lung cancer: a double blind randomised clinical phase III trial (CYCLUS study) by the Swedish Lung Cancer Study Group. Eur J Cancer 47: 1546-1555, 2011.

36. Holcik M, Yeh C, Korneluk RG and Chow T: Translational upregulation of $\mathrm{X}$-linked inhibitor of apoptosis (XIAP) increases resistance to radiation induced cell death. Oncogene 19: 4174-4177, 2000

37. Wilkinson JC, Cepero E, Boise LH and Duckett CS: Upstream regulatory role for XIAP in receptor-mediated apoptosis. Mol Cell Biol 24: 7003-7014, 2004.

38. Pan Q, Liu B, Liu J, Cai R, Liu X and Qian C: Synergistic antitumor activity of XIAP-shRNA and TRAIL expressed by oncolytic adenoviruses in experimental HCC. Acta Oncol 47: $135-144,2008$.

39. Ghosh SK, Yigit MV, Uchida M, et al: Sequence-dependent combination therapy with doxorubicin and a survivin-specific small interfering RNA nanodrug demonstrates efficacy in models of adenocarcinoma. Int J Cancer 134: 1758-1766, 2014.

40. Du Y, Shi A, Han B, et al: COX-2 silencing enhances tamoxifen antitumor activity in breast cancer in vivo and in vitro. Int $\mathrm{J}$ Oncol 44: 1385-1393, 2014.

41. Xue H, Chen Y, Cai X, et al: The combined effect of survivintargeted shRNA and emodin on the proliferation and invasion of ovarian cancer cells. Anticancer Drugs 24: 937-944, 2013.

42. Zhang Q, Meng X, Zheng G, et al: Antitumor activity of celecoxib, a selective cyclooxygenase- 2 inhibitor, in medullary thyroid carcinoma. Mol Med Rep 9: 768-772, 2014.

43. Vivanco I and Sawyers CL: The phosphatidylinositol 3-kinase AKT pathway in human cancer. Nat Rev Cancer 2: 489-501, 2002.

44. Perez-Tenorio G, Stal O and Southeast Sweden Breast Cancer Group: Activation of AKT/PKB in breast cancer predicts a worse outcome among endocrine treated patients. Br J Cancer 86: $540-545,2002$ 